\title{
Littérature, féminisme et connaissance de langues : à propos d'Emilia Pardo Bazán
}

\author{
Juan Francisco García Bascuñana
}

\section{(2) OpenEdition \\ Journals}

Édition électronique

URL : https://journals.openedition.org/dhfles/3166

DOI : $10.4000 /$ dhfles.3166

ISSN : 2221-4038

Éditeur

Société Internationale pour l'Histoire du Français Langue Étrangère ou Seconde

Édition imprimée

Date de publication : 1 juin 2012

ISSN : $0992-7654$

Référence électronique

Juan Francisco García Bascuñana, « Littérature, féminisme et connaissance de langues : à propos d'Emilia Pardo Bazán », Documents pour l'histoire du français langue étrangère ou seconde [En ligne], 47-48 | 2012, mis en ligne le 01 janvier 2015, consulté le 25 mars 2023. URL : http:// journals.openedition.org/dhfles/3166; DOI : https://doi.org/10.4000/dhfles.3166

Ce document a été généré automatiquement le 25 mars 2023

Tous droits réservés 


\title{
Littérature, féminisme et connaissance de langues : à propos d'Emilia Pardo Bazán ${ }^{1}$
}

\author{
Juan Francisco García Bascuñana
}

\section{Introduction}

1 Notre étude prétend mettre en évidence un cas concernant la figure de l'écrivaine espagnole Emilia Pardo Bazán (1851-1921), en soulignant l'un des aspects les moins connus de sa biographie : son rapport avec les langues étrangères et plus exactement avec le français. On connaît bien son œuvre de romancière vivement intéressée au naturalisme français qu'elle essaie de suivre dans ses écrits mais en l'adaptant à la réalité espagnole ; on ne connaît pas moins ses positions féministes qui débouchent, au fil des ans, sur une militance avérée pour les droits des femmes, et cela malgré ses convictions religieuses qui la portent souvent à des situations contradictoires. En tout cas, c'est pour dire l'obsession qui l'accompagne tout le long de sa vie et de son œuvre, liée inextricablement à ces élans d'émancipation féminine, à son avis l'un des signes inéluctables de la modernité. Mais il y a aussi un autre signe porteur de modernité qu'elle saura mettre au jour : l'importance de connaître des langues étrangères. Et elle donnera l'exemple, car elle ne se contentera pas de maitriser le français, une langue qu'elle avait apprise dans une «école française » de Madrid, fréquentée par des filles de l'aristocratie madrilène et dont elle ne garda pas, comme on le verra par la suite, un très bon souvenir. Et elle apprendra plus tard aussi, plus ou moins bien, l'anglais, l'allemand et l'italien², et encouragera ses compatriotes à l'imiter.

2 Nous allons donc faire ressortir dans notre étude un aspect qui nous semble essentiel: cette recherche de modernité de la part de Pardo Bazán se fait sous le signe de ce double engagement que constituent bien sûr la littérature et le féminisme, mais en soulignant son intérêt pour les langues vivantes, ce qui sert à expliquer mieux la riche personnalité de notre écrivaine et à la placer plus convenablement dans son temps. 
3 Et c'est précisément pour la situer dans son temps, et surtout à ce moment précis où elle commence sa première relation avec une langue étrangère, en l'occurrence le français, que nous allons faire un saut en arrière et nous situer aux années cinquante du XIX ${ }^{\mathrm{e}}$ siècle. Emilia Pardo Bazán, qui était née à La Corogne en 1851, commencera à fréquenter, à l'âge de six ans, une école française de Madrid où elle restera quelques années. Nous voilà donc plongés dans une période incontournable concernant l'enseignement du français en Espagne. Car ces années, on le sait bien, représentent un point d'inflexion dans une longue histoire de près de trois siècles.

\section{L'éducation des filles et les institutions scolaires espagnoles vers 1857}

4 Pour ne signaler que le détail le plus frappant, nous voulons rappeler ici que la publication de manuels pour l'enseignement du français s'élargit alors, vers ces années charnières du XIX ${ }^{e}$ siècle, d'une manière considérable. En dix ans, entre 1850 et 1860, on dénombre plus d'une trentaine de nouveaux manuels (Fischer, García Bascuñana \& Gómez 2004: 247-249) mis à la disposition des nouvelles générations d'étudiants espagnols qui veulent apprendre le français. Et l'une des conséquences les plus évidentes de la nouvelle réalité éducative qui commence à s'imposer pendant cette décennie, c'est précisément la promulgation de la loi Moyano, une loi qui, comme le signale avec pertinence Carmen Roig (1994: 40) a constitué « le premier effort sérieux du gouvernement espagnol pour instaurer un enseignement secondaire digne de ce nom ». Cette loi générale d'Instruction publique, votée en 1857, n'est que le résultat "naturel» d'une situation sociale et politique qui prend forme alors. Mais si nous tournons notre regard vers elle c'est surtout parce qu'elle est aussi inextricablement liée à l'enseignement des langues étrangères et tout spécialement du français. C'est pour dire l'intérêt de cette décennie dans laquelle «par hasard " Emilia Pardo Bazán commence à apprendre le français. C'est alors, en septembre 1857, précisément le mois où s'approuve la loi d'instruction publique, que la petite Emilia arrive à Madrid pour suivre des cours dans un pensionnat tenu par une dame française, Madame Lévy, qui était d'ailleurs la professeure de français de l'infante Isabelle, fille aînée de la reine Isabelle II. Cela faisait que cette école/pensionnat jouissait de la protection de la Maison royale. Eva Acosta, auteur d'une belle biographie de l'écrivaine galicienne raconte de façon détaillée l'instant précis du début de l'automne 1857 où la famille d'Emilia - une famille aisée appartenant à la petite noblesse provinciale - va décider, à propos de ses études, le chemin à suivre :

En 1857, la loi de Claudio Moyano établit l'éducation obligatoire pour garçons et filles, une décision qui mettra longtemps à se généraliser. Dans les villes les garçons de la bourgeoisie fréquentent l'école, mais le cas des filles est bien différent. À celles qui reçoivent une éducation formelle - une minorité - on offre deux chemins. Les " parents vieux-jeu » leur font apprendre, dans le meilleur des cas, à lire, à écrire et les quatre opérations arithmétiques de base, et ils les élèvent dans la réclusion la plus absolue. Quant à ceux qui se vantent de faire étalage du «vernis français en vogue ", ils emmènent leurs filles dans un pensionnat dirigé par une dame française, où au lieu d'apprendre les arts domestiques on leur fait prendre « des habitudes de duchesse millionnaire ». D'une part, un demi-analphabétisme bigot et rustre; de l'autre, un demi-analphabétisme prétentieux (Acosta 2007: 32) [traduction de JGB]. 
Ces mots spécialement pertinents vont donc nous servir pour rentrer dans le vif de notre sujet. C'est-à-dire que nous allons nous interroger surtout à propos de l'enseignement $d u$ français, pendant cette décennie toute particulière, mais surtout nous allons nous intéresser aux rapports des femmes avec cet enseignement, et on va le faire par le biais de cette figure d'exception qu'est Emilia Pardo Bazán. Car quand on parle de l'enseignement du français en Espagne, on ne tient pas toujours compte de ces fillettes et de ces jeunes filles, appartenant surtout, à quelques exceptions près, à des familles de l'aristocratie et de la haute bourgeoisie, qu'on mettait à apprendre le français, en tant qu'ornement indispensable pour toute femme qui se prisait alors. Bien sûr la langue française n'était pas le seul «ornement » de ce " programme d'études » réservé à ces filles de bonne famille fréquentant ces écoles/pensionnats français qu'on commençait alors à ouvrir dans la capitale du royaume et parmi lesquels celui de Madame Lévy aurait été l'un des plus réputées. Il y avait, bien sûr, d'autres disciplines à part le français qu'on jugeait aussi nécessaires pour former ces demoiselles qui devaient devenir un jour, pour le dire avec un mot de l'époque, «les anges du foyer ». Dans l'école de Madame Lévy, où Emilia avait été placée comme demi-pensionnaire ses parents s'étant installés à cette époque à Madrid -, elle pratique des travaux d'aiguille et le dessin, puis elle y apprend et pratique la musique, et étudie des matières telles que l'urbanité, des notions de géographie et de mythologie; sans oublier l'instruction religieuse qu'on considère indispensable pour la "formation intégrale " des demoiselles, le tout "pratiqué en français». C'est-à-dire toute une série d'«ornements» qui doivent faire partie du "plan d'études» des jeunes filles, sans qu'elles tombent pour autant dans l'odieuse catégorie de "bachelière ", une catégorie jugée peu convenable pour des demoiselles de bonne famille (Acosta 2007 : 34). Tout compte fait, le nouvel enseignement secondaire prôné par la loi Moyano n'était réservé finalement, malgré les « bonnes intentions » des législateurs, qu'aux garçons issus de la bourgeoisie ou de la petite bourgeoisie provinciale. Il s'opérait donc ainsi une double discrimination: l'une d'origine sociale et l'autre de genre. Plus évidente la première, plus subtile la seconde, puisqu'elle était acceptée, ou plutôt préconisée, tout naturellement par celles qu'on pourrait appeler les « classes dominantes ». De là vient qu'en principe on ne remet pas en question, dans les milieux aristocrates et bourgeois, cette nouvelle " éducation des filles ». Une éducation qui était nouvelle en Espagne et représentait malgré tout une avancée importante par rapport à la formation traditionnelle des femmes. En tout cas, elle aurait été remise en question plutôt parce qu'étrangère aux conventions sociales régnant jusqu'alors que pour les contenus mêmes de cette éducation qu'on considérait comme convenables.

\section{Souvenirs d'école}

6 Mais quels souvenirs gardera Emilia Pardo Bazán de ces années d'apprentissage chez Madame Lévy? Il faut dire qu'ils ne sont pas précisément bons. Elle les évoque avec indifférence et même avec dégoût, mais surtout avec une certaine ironie, dans la préface de la première édition (1886) de son roman le plus connu, Los pazos de Ulloa (Le château d'Ulloa, d'après sa traduction en français). Le portrait qu'elle fait de la directrice du pensionnat n'est pas précisément flatteur. Elle dit à son propos : «Elle nous traitait pis que des galériens. Elle nous donnait à manger un fricot immangeable et comme dessert des cacahouètes très rances, des noisettes toutes creuses et des marrons qui 
étaient de vrais fossiles, les pires qu'on pouvait acheter dans toute la ville » (Pardo Bazán 1886 : préface).

Mais au-delà de ses "mauvais souvenirs » des repas du pensionnat, nous voulons surtout tourner notre regard vers la relation de la petite Emilia avec son éducation proprement dite, pendant ces années dans l'école de Madame Lévy. Et surtout nous poser des questions sur sa première relation avec une langue étrangère, c'est-à-dire avec le français, et nous intéresser aux livres qui lui servaient à apprendre cette langue. Sur ce point, elle va se plaindre, en principe, des textes utilisés dans son école: "Telémaco por activa y por pasiva, Fábulas de Lafontaine a pasto » [Du Télémaque dans tous les sens, des Fables de Lafontaine à foison] (Pardo Bazán 1886: préface). Mais malgré cela, elle doit admettre finalement que cette relation constante avec des textes français n'a pas été après tout négative. Car les lectures françaises, suivies d'une pratique qu'on peut appeler grammatico-littéraire, s'accompagnent en plus de l'obligation de parler français pendant les heures passées à l'école, aussi bien dans la salle de classe qu'au réfectoire ou dans la cour de récréation, ce qui va rendre plus facile l'apprentissage de la nouvelle langue. Pardo Bazán le dit avec des mots teints d'ironie mais en même temps sincères: "...comme on nous interdisait de parler espagnol, les moins sottes d'entre nous, en sortant de là, étions devenues de vrais perroquets qui ne cessaient de parler français » (Pardo Bazán 1988 : préface).

Toutefois une question se pose quand notre écrivaine évoque plus tard ces années de son école française. Car s'il est vrai que, comme on l'a déjà dit, elle ne gardera pas un très bon souvenir de ce séjour chez Madame Lévy, on peut se demander si à la longue cette expérience a été si négative. Lire certains auteurs français dans leur langue originale et travailler leurs textes, même si cela, en principe, semble une tâche monotone et peu agréable pour une petite fille d'à peine sept ou huit ans, va l'aider, bien sûr, à maîtriser la langue française et à entrer en contact très tôt avec sa littérature.

\section{Lectures françaises}

Elle aura beau se plaindre de quelques-unes des lectures de son pensionnat français, elles vont lui servir à pouvoir "aller plus loin", car c'est là qu'elle apprendra à connaître l'œuvre littéraire de La Fontaine, de Fénelon et de Racine, entre autres. Sans ces lectures des auteurs classiques, on se demande si elle aurait été préparée pour profiter plus tard des grands auteurs romantiques et surtout des romanciers naturalistes. Car sa familiarité avec les grands auteurs français la portera tout naturellement vers Balzac, George Sand, Hugo, Flaubert, et surtout Zola et les frères Goncourt, sans oublier le roman populaire d'Eugène Sue. Elle s'intéresse spécialement à Edmond Goncourt, qu'elle a connu personnellement et sur lequel elle jette son regard féminin au moment de faire l'éloge de l'homme et de l'œuvre. Elle souligne surtout la présence de la femme qu'elle découvre avec enthousiasme dans un bon nombre des œuvres des deux frères.

10 À une époque où s'impose la traduction des grands auteurs français, Pardo Bazán sera donc capable de les lire directement, sans intermédiaires. Bien sûr, elle a tout son droit de ne pas apprécier les Aventures de Télémaque, un livre qui va devenir la cible des attaques concernant ses lectures dans le pensionnat français. Mais on voudrait connaître les raisons exactes de cette aversion envers le livre de Fénelon dont elle nous 
dit qu'elle le trouvait "trop académique et ennuyeux ", et cela aussi bien pendant ses années scolaires que des années après (Pardo Bazán 1886 : préface). La raison de cette mésestime pour le Télémaque vient de l'époque même où la petite Emilia a eu ses premiers contacts avec ce livre. Probablement parce que dans les années 1850, le livre où Fénelon narrait les aventures du fils d'Ulysse " n'était plus en vogue »; ce livre avait désormais quelque chose de suranné, de démodé. Après cent cinquante ans de présence constante - très souvent comme manuel scolaire pour l'apprentissage de la langue française - dans un bon nombre de pays européens où le livre de l'archevêque de Cambrai était devenu un point de repère culturel incontournable, il avait commencé à perdre sa place d'honneur jusqu'à devenir un outil littéraire et linguistique auquel on avait recours par habitude et par routine et qui finissait par provoquer l'ennui et la lassitude surtout quand on l'utilisait comme «manuel de classe». On était donc bien loin du temps où les Aventures de Télémaque éveillaient l'intérêt de toutes sortes de lecteurs, y compris les plus petits, comme cela avait été le cas de l'écrivain José María Blanco White, qui avait découvert le livre de Fénelon à l'âge de sept ans et dont, d'après son propre aveu, il était parvenu à apprendre certains passages par cœur. La lecture du livre de Fénelon avait mis devant les yeux de l'écrivain hispano-anglais le monde splendide et brillant des dieux païens, un monde qui avait fini par le fasciner et qui l'obligeait même à se poser des questions inquiétantes pour un croyant (Blanco White, 1845 : 18). Que s'était-il passé soixante-dix ans après pour que ce même livre qui éveillait les sens et l'imagination du petit Blanco White ennuyât mortellement la petite Emilia? La réponse est évidente: à partir de la seconde moitié du XIX ${ }^{\mathrm{e}}$ siècle le Télémaque suscitait de moins en moins l'intérêt des lecteurs et des élèves qui essayaient d'apprendre le français par l'intermédiaire des aventures du jeune fils d'Ulysse; et cela malgré l'insistance des professeurs et des maîtres qui s'en servaient dans leurs cours. Mais quelle était la cause exacte de cette désuétude ? Sans doute le Romantisme et tous les nouveaux courants littéraires et culturels du XIX ${ }^{e}$ siècle y étaient-ils pour quelque chose. Martín de Riquer et José María Valverde essayent d'expliquer dans un passage de leur Historia de la literatura universal les raisons du changement opéré à l'égard du Télémaque, tout en se montrant spécialement sévères envers le livre de Fénelon:

Le jeune Télémaque et le raisonnable Mentor [...] sont les protagonistes d'une ennuyeuse narration [...] qui eut un succès si fabuleux et incompréhensiblement durable que nos grands-parents, lorsqu'ils apprenaient le français, furent obligés encore d'endurer la dure épreuve de lire ses pages interminables. Car Les aventures de Télémaque, qui révoltent et indignent ceux qui ont lu l'odyssée, est un livre de circonstance et appartient à une époque précise, une œuvre qui cessa d'avoir du sens lorsque la Révolution française éclata. (Riquer \& Valverde 1968: II, 375) [traduction de JGB]

11 Tout compte fait ces grands-parents dont parlent Riquer et Valverde étaient, à quelques années près, les contemporains de Pardo Bazán qui, probablement, sentait aussi le même ennui devant un livre qui avait cessé alors d'être un modèle à suivre, capable d'éblouir des générations successives de lecteurs et de lectrices. À l'époque où Emilia fréquentait son pensionnat madrilène, l'heure du Télémaque de Fénelon était donc passée en tant qu'outil pédagogique et didactique.

12 En tout cas, au-delà du rejet apparent de Pardo Bazán de ce livre qu'on lui faisait lire sans cesse, on devrait s'interroger à propos de l'usage qu'on en faisait dans le pensionnat-école de Madame Lévy, tout en se posant une question au préalable qui nous semble spécialement pertinente : quelle aurait été vraiment la cause du manque d'empathie de l'écrivaine galicienne avec le livre de Fénelon? D'aucuns pourraient 
proposer comme cause principale de ce rejet - on pourrait discuter si c'est avec raison le contenu même d'un livre considéré en principe comme " éminemment féminin ", où une certaine sensibilité imprégnait la plupart de ses longs passages, sans pour autant cesser d'être un livre où les voix masculines se faisaient trop entendre. Mais alors, pourrait-on se demander, pourquoi donc la petite Emilia, presque vers la même époque où elle étudiait dans cette école française de Madrid, lisait-elle avec enthousiasme des histoires sur la conquête de l'Amérique, comme, par exemple, l'Historia verdadera de la conquista de la Nueva España de Bernal Díaz del Castillo où pratiquement tous les grands protagonistes étaient des hommes? Il faudrait voir plutôt dans ce rejet un refus d'un certain type d'éducation qu'elle jugeait trop discriminatoire pour les femmes. C'est ce qu'elle nous raconte aussi, dans la note autobiographique qui précède la première édition de Los pazos de Ulloa, à propos de ses premières leçons de piano, un autre des ornements jugés nécessaires pour compléter la formation des demoiselles de bonne famille. Elle nous dit qu'elle s'ennuyait à mourir pendant ces moments interminables devant le piano et enviait ses cousins qui à ce même instant recevaient des cours de latin, comme il convenait alors aux garçons. La conclusion qu'elle tirait de cette situation était claire: apprendre le latin devait être très important puisqu'on le réservait aux hommes, tandis que l'apprentissage du piano ne devait pas beaucoup compter car on le destinait presque exclusivement aux femmes. C'est donc dans cette même perspective qu'il faut envisager la lecture et l'utilisation du Télémaque à l'école de Madame Lévy et d'emblée l'apprentissage même de la langue française. De toute façon, et malgré ses critiques plus ou moins explicites, elle ne semble vraiment pas remettre en question cet enseignement qui l'a aidée somme toute à apprendre le français. Elle se vantera même de sa maîtrise de cette langue, qui aurait facilité plus tard son accueil dans les milieux littéraires et artistiques français pendant ses longs séjours parisiens ${ }^{3}$.

Toutefois, une question primordiale se pose à propos de la relation de Pardo Bazán avec la langue française. Car il faudrait savoir si les notes autobiographiques de 1886, lorsque l'écrivaine a déjà trente-cinq ans et que ses livres commencent à être connus, répondent vraiment à de vrais souvenirs d'enfance ou s'il s'agit plutôt des souvenirs reconstitués, fruits plutôt de ses expériences postérieures de jeune fille et de femme adulte. Sa façon d'envisager sa relation avec les lectures littéraires utilisées pour l'apprentissage du français semblent plutôt une recréation a posteriori, un travail de mémoire sélective que la reproduction exacte de ses souvenirs. En tout cas, et pour revenir au Télémaque, il faut admettre que le manque d'empathie avec ce livre proviendrait plutôt des découvertes littéraires qu'elle a faites à partir de sa jeunesse où le livre de Fénelon ne pourra pas résister, vu les goûts de Pardo Bazán, à la comparaison avec les romans qui surgissent des nouveaux courants littéraires auxquels elle s'intéresse, tout spécialement le naturalisme. Pendant ses années d'enfance le Télémaque ${ }^{4}$ 'aurait donc été qu'un livre ennuyeux qu'on l'obligeait à lire, à mémoriser et à répéter pendant de longues heures, le tout accompagné d'exercices grammaticaux routiniers et excessivement répétitifs, un livre qui ennuyait profondément une fillette de moins de dix ans, qui, bien sûr, ne pouvait porter alors sur l'œuvre un jugement précis et fondé.

Par contre elle sera toujours moins sévère à l'égard des fables de La Fontaine et surtout de la Phèdre de Racine, les deux autres ouvrages qu'elle cite explicitement en parlant de l'enseignement du français dans son pensionnat madrilène. Il semble même qu'on lui faisait apprendre par cœur un bon nombre de fables qu'elle devait répéter pour corriger sa prononciation française. La fillette qu'elle était alors finissait par 
s'intéresser à ces fables où apparaissaient des animaux déambulant dans une campagne plus ou moins stéréotypée mais qui lui rappelait souvent le monde rural de sa Galice natale, où elle passait ses grandes vacances. Mais ce sont les vers de Phèdre qu'elle aimera tout spécialement, jusqu'à arriver même à en apprendre de longues tirades. Car la pièce de Racine servait aussi pour compléter les cours de mythologie, en lui expliquant - en français, bien sûr -, toutes ces «belles histoires » qui avaient comme protagonistes les dieux et les déesses, les héros et les héroïnes de l'ancienne Grèce. Le drame de Phèdre et des autres personnages de la pièce était certes incompréhensible pour une petite fille, mais la cadence des vers et les énormes possibilités didactiques que renferment toujours les textes dramatiques ne pouvaient qu'aider à l'apprentissage et parvenaient à créer une intense émotion et une empathie spontanée, plus ou moins consciente, avec le texte de Racine. Elle gardera donc un souvenir inoubliable de cette pièce et elle y reviendra plus tard en se rappelant certains des vers qu'elle avait appris chez Madame Lévy. Elle ne perdra pas l'occasion de le faire, n'importe quelle circonstance lui sert à réciter des tirades apprises et mémorisées longtemps auparavant. Même dans les moments les plus inattendus, ses souvenirs d'école affleureront sous forme de vers. C'est ainsi qu'en se voyant entourée d'une multitude qui l'empêche même de marcher, lors d'une visite à l'exposition universelle de Paris de 1889 , où elle était venue pour faire des chroniques pour des journaux espagnols et latino-américains, elle s'exclame en se souvenant des mots de Phèdre, à la scène III du premier acte de la pièce de Racine :

« Dieux! Que ne suis-je assise à l'ombre des forêts » (Pardo Bazán 2003 : 171).

\section{L'éducation des filles et l'apprentissage des langues étrangères}

15 Tout compte fait, cette " méthode directe » avant la lettre, un peu sui generis, à l'usage de ces filles qui fréquentaient l'école-pensionnat de Madame Lévy, aurait vraiment servi à Emilia Pardo Bazán. L'obligation de parler tout le temps en français, ces répétions de textes classiques, tout cela facilitera l'apprentissage de cette langue. On a beau critiquer cette méthode, la trouver inappropriée pour des fillettes qui n'avaient pas plus de douze ans, il faudra admettre néanmoins que dans le cas de notre écrivaine la "méthode " aurait bien réussi. La question serait de savoir si elle a servi aussi à ses compagnes d'école et à toutes les filles qui, à la même époque, fréquentaient d'autres écoles françaises qui ouvraient alors leurs portes à Madrid et dans d'autres grandes villes espagnoles. En tout cas, c'était un enseignement du français complètement différent de celui dont on se servait alors dans ces lycées d'enseignement secondaire créés après la loi générale d'instruction publique de 1857. Un enseignement, celui-ci éminemment traditionnel, fondé sur la grammaire et la traduction, comme le montrent les nombreux manuels publiés dans les années cinquante et soixante du siècle. Tous, au-delà de leurs titres et de leurs préfaces remplis de bonnes intentions, présentent le français, à quelques exceptions près, d'une façon tout à fait traditionnelle ou répètent le Chantreau dans tous les sens, malgré les adjectifs pompeux qui les accompagnent ("nuevo Chantreau», "novísimo Chantreau » ou "Chantreau reformado »). Mais ce qu'il nous intéresse de souligner encore une fois, c'est que l'enseignement secondaire surgi de la loi Moyano est adressé presque exclusivement aux garçons, car la condition de bachelier n'était réservée qu'à ceux-ci ; mais évidemment pas à tous, à une époque où près de $75 \%$ de la population espagnole était analphabète. En tout cas, ce qui est 
évident c'est qu'il n'était pas convenable, vers 1850-1860, que les jeunes filles deviennent bachelières, une catégorie, comme on a dit plus haut, jugée peu convenable. Cette discrimination provoquera, d'ailleurs, une situation quelque peu curieuse : les jeunes filles de bonne famille auront une maîtrise du français, à laquelle leurs contemporains masculins ne parviendront presque jamais. C'est que, comme on l'a vu, la méthode d'enseignement du français était très différente pour les deux sexes. Pour elles, un bon apprentissage de cette langue devait être, en principe, un attribut précieux qui devait servir à augmenter les "atouts de la féminité », telle qu'on l'entendait alors dans certains milieux aristocratiques et bourgeois; tandis que pour eux le français n'était qu'une des matières de leur programme d'étude, qui ne comptait pas même parmi les plus importantes (Fernández Fraile \& Suso 1999 : 197-220).

Ainsi donc l'apprentissage des langues deviendra pour Pardo Bazán, contrairement à ce qu'on avait prévu dans le pensionnat élitiste de Madame Lévy, l'un de ces atouts qu'elle considère comme indispensables pour une bonne formation intégrale et qui sont inextricablement liés d'ailleurs aux élans d'indépendance et d'émancipation féminine ${ }^{5}$, un signe porteur de modernité qu'elle saura mettre au jour, surtout après ses voyages à l'étranger; en France, pays qu'elle connaissait bien évidemment et où elle fit de longs séjours, mais aussi dans les différents pays européens qu'elle a visités (l'Angleterre, l'Allemagne, l'Autriche, la Suisse, la Belgique ou l'Italie). Car après le français elle s'efforcera aussi d'apprendre l'anglais et l'allemand, sans compter l'italien et le portugais qu'elle parlait et comprenait sans problème. Mais quoi qu'on dise, son apprentissage de ces autres langues en plus du français ne fut pas complètement autodidacte. Elle ne les aurait pas apprises seulement grâce à ses lectures, comme on le suggère souvent mais, d'après son propre aveu - pour ce qui concerne en tout cas l'apprentissage de l'anglais -, elle reçut des leçons de cette langue de la part d'un professeur londonien, "qui était très fier de l'être et qui se moquait de moi avec piquant en apprenant mes projets ambitieux de traduire d'ici peu la scène de la mort de Desdémone ou le Farewell de Byron » (Freire López 2001 : 310-311). Quant à l'allemand, bien qu'on n'ait pas de témoignages précis de la façon comment elle l'apprit, on doute que ce fût seulement à travers des lectures. De toute façon, ses traductions de la poésie de Heinrich Heine montre qu'elle devait avoir une bonne connaissance de cette langue, même si d'aucuns en doutent et suggèrent qu'elle aurait pu la traduire moyennant le français, une pratique assez courante à l'époque où le français devenait souvent une langue intermédiaire (Freire López 2009: 864-865). Reste à savoir quelle était sa connaissance exacte de la langue courante, bien que les témoignages de ses voyages en Allemagne et en Autriche ${ }^{6}$ montreraient qu'elle devait avoir une certaine maîtrise de cette langue.

17 Cet enthousiasme pour les langues étrangères poussera donc notre écrivaine à encourager ses compatriotes à la suivre sur ce chemin pour mieux partager avec les autres leurs inquiétudes et leurs aspirations, et les imiter si nécessaire. Car tout au long des pages de ses livres de voyage (Pardo Bazán 2003) la romancière galicienne nous apparaît comme une personne spécialement intéressée aux différentes cultures des pays européens et spécialement au rôle que les femmes y jouent ${ }^{7}$. 


\section{Conclusion}

Son ancienne expérience d'étudiante de français aurait servi, somme toute, à Emilia Pardo Bazán pour s'approcher plus facilement d'autres langues. Pour ce faire, sa méthode d'apprentissage sera spécialement simple et directe: les lectures et les voyages seront des instruments dont elle se servira pour augmenter son bagage plurilinguistique. D'une certaine façon, elle devra admettre que l'apprentissage dans son ancien pensionnat madrilène, fondé surtout sur des lectures de textes classiques, «n'était pas si bête »; ce qu'il fallait désormais, après son expérience scolaire, c'était de bien savoir choisir les auteurs. Et cet élan la poussera donc tout naturellement vers d'autres langues, car elle souhaitait surtout pouvoir lire les grands écrivains en leur langue originale et connaitre mieux cette Europe qui la fascinait, ce qui lui semblait impossible sans la connaissance de la langue de chacun des pays qu'elle visitait ${ }^{8}$.

Elle «a pu faire » finalement tout ce qu'elle souhaitait, aussi bien comme femme que comme écrivaine, et elle est parvenue même à rendre compatible « la pratique du piano et l'apprentissage du latin ", ce qui lui semblait impossible à l'âge de dix ans, étant donné sa condition féminine ${ }^{9}$. À une époque où il n'existait ni les quotas paritaires ni la discrimination positive, Pardo Bazán fut un exemple incontestable de ce que signifiait vraiment l'égalité des sexes. Elle s'efforça d'échapper à la condition qui lui était réservée, sans doute meilleure que celle à laquelle étaient vouées la plupart des femmes espagnoles de son temps, mais tout compte fait marquée du sceau de la soumission féminine.

\section{BIBLIOGRAPHIE}

ACOSTA, Eva (2007). Emilia Pardo Bazán : la luz en la batalla. Biografía. Barcelona : Lumen.

BLANCO WHITE, José María (1845). The Life of the rev. Joseph Blanco White; written by himself with portions of his correspondance. 3 v. London : John Chapman, v. 1.

FERNÁNDEZ FRAILE, María Eugenia \& SUSO, Javier (1999). La enseñanza del francés en España, 1767-1936 : estudio histórico, objetivos, contenidos y procedimientos. Granada : Método.

FISCHER, Denise, GARCÍA BASCUÑANA, Juan F. \& GÓMEZ, María Trinidad (2004). Repertorio de gramáticas y manuales para la enseñanza del francés en España (1565-1940). Barcelona : PPU.

FREIRE LÓPEZ, Ana María (2001). « La primera redacción, autógrafa e inédita de los Apuntes autobiográficos de Emilia Pardo Bazán », Cuadernos para la investigación de la literatura hispánica, XXVI, 305-336.

FREIRE LÓPEZ, Ana María (2009). «Emilia Pardo Bazán», in F. Lafarga \& L. Pegenaute éd. Diccionario histórico de la traducción en España. Madrid : Gredos, 864-866.

PARDO BAZÁN, Emilia (1886). Los pazos de Ulloa, [précédé d'] « Apuntes autobiográficos ».Barcelona : Daniel Cortezo.

PARDO BAZÁN, Emilia (2003). Viajes por Europa. Madrid : Bercimuel. 
RIQUER, Martín \& VALVERDE, José María (1968). Historia de la literatura universal. Barcelona :

Planeta, 3 vols.

ROIG, Carmen (1994). « La formation à l'enseignement du FLE en Espagne », Études de Linguistique Appliquée, 95, 40-47.

\section{NOTES}

1. Cet article a été élaboré et rédigé dans le cadre du projet de recherche FFI2008-0239/FILO financé par le ministère de la Science et de l'innovation espagnol.

2. Sans compter le portugais, qu'elle ne considérait pas véritablement comme une langue étrangère et qu'elle apprit de bonne heure grâce à sa connaissance du galicien de sa province natale.

3. Voir les livres Al pie de la torre Eiffel (117-281) et Por Francia y por Alemania inclus conjointement avec Mi Romería et Por la Europa católica dans une nouvelle édition sous le titre Viajes por Europa (Pardo Bazán 2003).

4. Chez Madame Lévy on travaillait exclusivement avec un original français, sans avoir recours à aucune des nombreuses traductions espagnoles du Télémaque.

5. Et il arrivait même qu'une éducation prévue pour former de futures dames, compagnes parfaites des hauts personnages de l'aristocratie ou de la bourgeoisie, pouvait servir à former des jeunes filles indépendantes. Celles qui devaient se limiter à être des femmes « obéissantes et bien élevées" devenaient paradoxalement - la lecture y était pour quelque chose - des femmes inquiètes et peu soumises, capables de prendre leur destin en main.

6. Elle voyage à Vienne pour assister à l'exposition universelle de 1873.

7. Ce qui la portera, par exemple, à rédiger la préface de la traduction espagnole de The Subjection of Women de John Stuart Mill.

8. La question se pose vraiment à propos du niveau de connaissance de chacune de ces nouvelles langues étrangères qu'elle a apprises, car malgré ses bons propos, et étant donné que les circonstances d'apprentissage n'étaient pas toujours les mêmes, elle ne parviendra jamais à en avoir une maîtrise semblable à celle du français, surtout pour ce qui concerne la langue parlée. Mais elle deviendra pour autant une bonne lectrice en anglais, en allemand et en italien doublée d'une bonne traductrice aussi -, ce qui n'était pas alors un moindre détail.

9. Ce n'est pas par hasard qu'elle deviendra en 1916 titulaire d'une chaire de littératures romanes à l'université de Madrid. Elle sera la première femme à occuper cette place dans une université espagnole, au grand scandale de la société de son temps.

\section{RÉSUMÉS}

On connaît bien les romans d'Emilia Pardo Bazán, tournés en partie vers le naturalisme français. On ne connaît pas moins les positions féministes de cette écrivaine qui débouchent sur une militance pour les droits des femmes. Mais il y a aussi un signe porteur de " modernité » qu'elle saura mettre à jour : l'importance de connaître des langues étrangères. Et elle donnera l'exemple, car elle ne se contentera pas de maîtriser le français, une langue qu'elle avait apprise dans une « école française » de Madrid fréquentée par des filles de l'aristocratie. Elle apprendra aussi plus 
tard l'anglais et l'allemand, et encouragera ses compatriotes à la suivre pour mieux partager avec les autres leurs inquiétudes et leurs aspirations et les imiter si nécessaire. Nous soulignons donc la relation étroite de Pardo Bazán avec les langues étrangères, mais en nous intéressant surtout aux méthodes et aux livres qu'elle utilisait pour l'apprentissage du français.

We are familiar with the novels of Emilia Pardo Bazán, which bear a certain resemblance to French Naturalism. Her feminist views, which led her to fight for the rights of women, are equally well known. However, she also displays a feature of "modernity" that we shall attempt to shed light on here: she attached considerable importance to foreign languages. She preached by example and, not being satisfied merely with the French that she had learned at a French school in Madrid attended by young girls from aristocratic families, she also went on to learn English and German. She encouraged her fellow compatriots to follow her example, she shared the concerns and aspirations of others, and she imitated them if necessary. We focus, then, on the close relation between Pardo Bazán and foreign languages, and take particular interest in the methods and books she used to learn French.

\section{INDEX}

Keywords : Women, Feminism, Teaching of French, Knowledge of languages, Literature, Reading. Mots-clés : Femmes, Féminisme, Enseignement du français, Connaissance de langues, Littérature, Lecture.

\section{AUTEUR}

\section{JUAN FRANCISCO GARCÍA BASCUÑANA}

Universitat Rovira i Virgili - Tarragone 